\title{
Osteopontin regulates proliferation, apoptosis, and migration of murine claudin-low mammary tumor cells
}

\author{
S. Saleh, D. E. Thompson, J. McConkey, P. Murray and R. A. Moorehead
}

\begin{abstract}
Background: Osteopontin is a secreted phosphoglycoprotein that is expressed by a number of normal cells as well as a variety of tumor cells. With respect to breast cancer, osteopontin has been implicated in regulating tumor cell proliferation and migration/metastasis and may serve as a prognostic indicator. However it remains unclear whether osteopontin has the same impact in all breast cancer subtypes and in particular, osteopontin's effects in claudin-low breast cancer are poorly understood.

Methods: CDNA microarrays and qRT-PCR were used to evaluate osteopontin expression in mammary tumors from MTB-IGFIR transgenic mice and cell lines derived from these tumors. siRNA was then used to determine the impact of osteopontin knockdown on proliferation, apoptosis and migration in vitro in two murine claudin-low cell lines as well as identify the receptor mediating osteopontin's physiologic effects.

Results: Osteopontin was expressed at high levels in mammary tumors derived from MTB-IGFIR transgenic mice compared to normal mammary tissue. Evaluation of cell lines derived from different mammary tumors revealed that mammary tumor cells with claudin-low characteristic expressed high levels of osteopontin whereas mammary tumor cells with mixed luminal and basal-like features expressed lower levels of osteopontin. Reduction of osteopontin levels using siRNA significantly reduced proliferation and migration while increasing apoptosis in the claudin-low cell lines. Osteopontin's effect appear to be mediated through a receptor containing ITGAV and not through CD44.

Conclusions: Our data suggests that mammary tumors with a mixed luminal/basal-like phenotype express high levels of osteopontin however this osteopontin appears to be largely produced by non-tumor cells in the tumor microenvironment. In contrast tumor cells with claudin-low characteristics express high levels of osteopontin and a reduction of osteopontin in these cells impaired proliferation, survival and migration.
\end{abstract}

Keywords: Osteopontin, Breast cancer, Claudin-low, Proliferation, Apoptosis, Migration

\section{Background}

Osteopontin (OPN, Spp1) is a secreted glycophosphoprotein expressed by a number of cell types including endothelial cells, vascular smooth muscle cells, neural cells, epithelial cells, osteoblasts/osteoclasts, and immune cells [1-3]. OPN is involved in normal processes including wound healing, bone remodelling and inflammation as well as pathological processes such as cancer [4-7]. OPN mediates its actions through binding to

\footnotetext{
* Correspondence: rmoorehe@uoguelph.ca

Department of Biomedical Science, Ontario Veterinary College, University of Guelph, 50 Stone Road East, Guelph, ON N1G2W1, Canada
}

integrins including $\alpha \mathrm{v} \beta 1, \alpha \mathrm{v} \beta 3, \alpha \mathrm{v} \beta 5, \alpha \mathrm{v} \beta 6, \alpha 4 \beta 1, \alpha 5 \beta 1$, $\alpha 8 \beta 1$, and $\alpha 9 \beta 1$ as well as CD44 [8-10].

In the mid-1990's OPN mRNA and protein were found to be elevated in a number of different tumors compared to matching control tissue $[11,12]$. Elevated levels of OPN have been found in tumors of the breast, prostate, colon, ovary, stomach, lung and liver [13-22]. OPN has been observed both in tumor cells themselves and in stromal cells surrounding the tumor [23]. More recent studies have shown that OPN is also elevated in the serum of breast cancer patients including those with early stage disease [24]. In breast cancer, OPN has been 
associated with poor prognosis $[6,7]$ and OPN has been shown to increase breast cancer cell survival and migration [25-27]. OPN is found in ER positive breast cancer and triple negative tumors [28].

Murine mammary tumor models have also been used to examine OPN's role in breast tumorigenesis. A study investigating serum biomarkers in transgenic mice overexpressing an activated version of $c$-neu identified 3 proteins significantly elevated in tumor bearing mice compared to control mice and one of these proteins was OPN [29]. Interestingly, OPN was also able to discriminate tumor bearing mice from control mice when mammary tumor development was driven by a mutant p 53 protein [29]. The tumors induced by the mutant p53 protein were estrogen receptor positive while the tumors induced by $c$-neu expression were estrogen receptor negative suggesting that OPN is elevated in mammary tumors with diverse characteristics [29].

In our mouse mammary tumor model, MTB-IGFIR transgenic mice develop mammary tumors due to elevated expression of the type I insulin-like growth factor receptor (IGF-IR) in mammary epithelial cells [30]. The mammary tumors that arise in this model have characteristics of human luminal breast cancer including expression of cytokeratin 8, cytokeratin 18 and E-cadherin however, these tumors cluster most closely with human basal-like breast cancer when gene expression profiles are used [31, 32]. Expression of the IGF-IR transgene in the MTB-IGFIR mice is controlled by a doxycycline inducible promoter and thus the impact of the loss of transgene expression in established mammary tumors can be evaluated. Loss of IGF-IR transgene expression in mammary tumors promotes regression followed by tumor re-growth in a subset of the mice. Mammary tumor recurrence in the absence of IGF-IR transgene expression is associated with epithelial to mesenchymal transition (EMT) [33] and tumors that cluster most closely with human claudin-low mammary tumors [31]. A number of cell lines have been generated from these tumors. RJ345 cells share characteristics with the luminal/basal like tumors while RJ348 and RM11A share characteristics with the claudin-low tumors [34, 35]

DNA microarray analysis comparing wild type mammary tissue to the mammary tumors revealed that Spp1 was the most differentially expressed genes; $S p p 1$ was elevated 77 -fold in the mammary tumors compared to normal mammary glands [31]. Spp1 expression remained high in mammary tumors that acquired a more mesenchymal phenotype compared to normal mammary glands. Therefore, the purpose of this study was to further characterize the function of OPN in mammary tumorigenesis using murine mammary tumor cell lines and siRNA-mediated knockdown of OPN and its receptors.

\section{Methods \\ Cell culture}

The RM11A, RJ348 and RJ345 murine mammary tumour cells were grown in Dulbecco's modified eagle medium (DMEM) (Life Technologies Inc., Burlington, $\mathrm{ON})$ containing the following supplements: $10 \%$ tetracycline-free fetal bovine serum (FBS) (Clontech, Mountain View, CA), $1 \mathrm{mM}$ sodium pyruvate, $10 \mathrm{mM}$ 4-(2-hydroxyethyl)-1-piperazineethanesulfonic acid (HEPES), $4 \mathrm{mM}$ glutamine, $2 \mathrm{mM}$ hydrocortisone, $5 \mu \mathrm{g} /$ $\mathrm{ml}$ estrogen, $5 \mu \mathrm{g} / \mathrm{ml}$ prolactin, $10 \mu \mathrm{g} / \mathrm{ml}$ EGF, $10 \mu \mathrm{g} / \mathrm{ml}$ insulin, $10 \mu \mathrm{g} / \mathrm{ml}$ doxycycline and $1 \%$ antibioticantimycotic (Life Technologies Inc., Burlington, ON). Cells were maintained at $37^{\circ} \mathrm{C}$ and $5 \%$ carbon dioxide.

\section{RNA extraction}

For tissue samples, flash-frozen tissues were homogenized using a handheld homogenizer in lysis/binding buffer from the mirVana miRNA Isolation kit (Life Technologies Inc., Burlington, ON, Canada). For cell lines, cells were washed twice in ice cold PBS and the lysis/binding buffer from the mirVana miRNA Isolation kit as added directly to the plate. The cells were scraped off using a cell scraper and the cell-buffer solution was then collected into $1.5 \mathrm{ml}$ Eppendorf tubes. RNA from tissue and cell lines was extracted following the manufacturer's protocol (enrichment for small RNAs was not performed). RNA was eluted with nuclease-free water and stored at $-80^{\circ} \mathrm{C}$.

\section{Quantitative Real-Time PCR}

RNA (500 ng) was reversed transcribed using iScript Reverse Transcription SuperMix (Bio-Rad Laboratories, Mississauga, ON, Canada) following the manufacturer's protocol. The cDNA was then amplified using qScript cDNA SuperMix (Quanta Biosciences, Gaithersburg, $\mathrm{MD})$ and the PrimePCR program on a CFX96 real-time PCR machine (Bio-Rad Laboratories, Mississauga, ON, Canada). All primers were purchased from Bio-Rad Laboratories (Mississauga, ON, Canada) and CFX Manager software v3.1 (Bio-Rad Laboratories, Mississauga, ON, Canada) was used to calculate expression levels and primer efficiencies. Primer efficiencies were as follows, Spp 1 - 104, Itgav - 101, Itgb3 - 99, Cd44 - 101, Hprt 105, and Ywhaz - 110. The expression of Spp1, Itgav, Itgb3 and $C d 44$ were determined relative to the housekeeping genes Hprt and Ywhaz which were previously been shown to be suitable from a panel of 14 potential housekeeping genes [36].

\section{Immunohistochemisty}

Tissue sections from formalin-fixed, paraffin-embedded mammary tumors were de-waxed with xylene and rehydrated in 2 changes each of $100 \%, 90 \%$ and $70 \%$ 
ethanol followed by incubation in PBS. Sections were blocked with $5 \%$ BSA in Tris-buffered saline containing $0.1 \%$ triton-X100 at room temperature for $30 \mathrm{~min}$. The sections were then incubated with the OPN antibody (Ab8448, Abcam Inc, Toronto, ON) at a 1:200 dilution in PBS overnight at $4{ }^{\circ} \mathrm{C}$. An anti-rabbit IgG (B7389, whole molecule) secondary was used at a 1:200 dilution in PBS for $1 \mathrm{~h}$ at room temperature (Sigma-Aldrich Canada Co, Oakville, ON). Sections were then stained with hematoxylin, dehydrated and mounted. Sections lacking the primary antibody were used as a control.

\section{Western blotting}

Western blotting was performed as previously described [30]. Anti-OPN (AKm2A1; Santa Cruz Technologies, Santa Cruz, MA) and anti- $\beta$-actin (Cell Signaling Technology Beverly, MA) were used at a 1:1,000 dilution in $5 \%$ skim milk in Tris-buffered Saline (TBS) containing $0.01 \%$ Tween 20 (TBST). An anti-mouse secondary was used for detection of OPN while an anti-rabbit secondary was used for the detection of $\beta$-actin. Both secondary antibodies were obtained from Cell Signaling Technology (Beverly, MA) and were used at a 1:2,000 dilution in $5 \%$ skim milk in TBST. Bands were visualized using Western Lightning Chemiluminescence substrate (Perkin Elmer, Wellesley, MA, USA) and a FluorChem 9900 gel documentation imaging system (Alpha Innotech, San Leandro, CA).

\section{Transient OPN Knockdown (siRNA)}

Cells were transfected with stealth RNAi oligonucleotides directed against Spp1, Cd44, Itgav or a guaninecytosine (GC) control sequence. All oligonucleotides were obtained from Life Technologies Inc. (Burlington, $\mathrm{ON}$, Canada) and were used at a final concentration of 100 nM. Cells were transfected using Lipofectamine 2000 transfection reagent and Opti-MEM media (both obtained from Life Technologies Inc., Burlington, ON, Canada). After $4 \mathrm{~h}$, fully supplemented media was added to the wells and cells were incubated at $37{ }^{\circ} \mathrm{C}$ with $5 \%$ carbon dioxide.

\section{OPN Neutralizing Antibody}

The mouse osteopontin neutralizing antibody (cat \#AF808) was purchased from R\&D Systems (Minneapolis, MN) and used at a concentration of $5 \mu \mathrm{g} / \mu \mathrm{l}$ or $10 \mu \mathrm{g} / \mu \mathrm{l}$. A goat-anti-chicken IgG secondary antibody (Life Technologies, Burlington, ON, Canada) was used at $5 \mu \mathrm{g} / \mathrm{ml}$ or $10 \mu \mathrm{g} / \mathrm{ml}$ and served as a control.

\section{Immunofluorescence}

Cells were plated onto sterile coverslips and were treated with either siRNA targeting OPN mRNA (described above) or an OPN neutralizing antibody (described above) for $48 \mathrm{~h}$. Cells were then washed with PBS and fixed for $1 \mathrm{~h}$ at room temperature in $10 \%$ buffered formalin. Coverslips with fixed cells were then washed with PBS and permeated with $0.1 \%$ Triton $\mathrm{X}$ in PBS for $10 \mathrm{~min}$ at room temperature. Fixed cells were then washed once again with PBS, blocked in $5 \%$ BSA for $10 \mathrm{~min}$, and then incubated overnight at $4{ }^{\circ} \mathrm{C}$ with the primary antibody. Primary antibodies were used at a 1:200 dilution and anti-Ki67 (Abcam, Cambridge, MA) or anti-phospho-histone H3 (Abcam, Cambridge, MA) were used to identify proliferating cells while anticleaved caspase 3 (Millipore, Etobicoke, ON, Canada) was used to identify apoptotic cells. Fluorescent secondary antibodies were used a 1:100 dilution (Life Technologies, Burlington, ON, Canada) at room temperature for $2 \mathrm{~h}$. Cells were then counterstained with 4',6-diamidino2-phenylindole (DAPI) (Sigma, Oakville, ON, Canada) and mounted using Prolong Gold mounting media (Invitrogen, Burlington, ON, Canada). Images were captured with an Olympus BX-61 fluorescent microscope and positively stained cells were counted manually from at least 4 different, randomly selected fields of the coverslip.

\section{Cell counting}

RJ348 cells were seeded in 6-well plates at a density of 200,000 cells per well. Four hours after seeding (RJ348 cells had attached to the plate at this point), RJ348 cells were treated with either siRNA targeting OPN mRNA (described above) or an OPN neutralizing antibody (described above) for $48 \mathrm{~h}$. At this point cells were trypsinized, stained with typran blue and counted using a hemocytometer.

\section{Scratch wound migration assay}

Cells where plated in 24-well culture dishes and grown to $\sim 70-80 \%$ confluency. Cells were then transfected with siRNA targeting Spp1, Cd44, or Itgav, or a guaninecytosine control sequence as described above. Twentyfour hours post siRNA transfection a scratch was induced using a pipette tip and images were captured immediately after scratch induction as well as $24 \mathrm{~h}$ or $48 \mathrm{~h}$ after scratch induction. Images were captured using an Olympus IX71 inverted microscope (Toronto, ON, Canada) and Q-capture software (Surrey, BC, Canada). Image J software [37] was used to quantify the percent wound closure.

\section{Boyden chamber assay}

Matrigel (Life Technologies, Burlington, ON, Canada) was diluted 1:6 in DMEM (Life Technologies, Burlington, ON, Canada) and $20 \mu \mathrm{l}$ of diluted matrigel was plated on to the upper compartment of a Falcon cell culture insert (cat \#353097; BD Bioscience, Mississauga, 
ON, Canada). Approximately $1 \times 10^{5}$ cells were cultured in $200 \mathrm{ul}$ of serum-free media in the upper compartment of the insert. The bottom well was filled with $300 \mathrm{ul}$ of media containing serum. Cells were cultured at $37{ }^{\circ} \mathrm{C}$ and $5 \%$ carbon dioxide for $20 \mathrm{~h}$. Media was then aspirated from the lower chamber and the bottom of the insert was fixed with $5 \%$ glutaraldehyde in $1 x P B S$, for 10 min, washed with water and stained with $0.5 \%$ toluidine blue staining solution $10-20 \mathrm{~min}$ at room temperature. The inner surface of the upper chamber was then wiped clean and cells that had migrated to the bottom of the insert were visualized using an Olympus IX71 inverted microscope (Toronto, ON, Canada) and Q-capture software (Surrey, BC, Canada). The number of cells on the bottom of the insert were counted manually.

\section{Statistics}

A paired student's $t$ test was used to compare means from the treated and control groups. Differences were considered to be significant at $p<0.05$.

\section{Results}

OPN expression in mammary tissue, mammary tumors and mammary tumor cell lines

A previous study from our lab described the DNA microarray analysis of wild-type mammary tissue (WT), primary mammary tumors (PMT) and recurrent spindle tumors (RST) generated in MTB-IGFIR transgenic mice [31]. PMTs were induced by the transgenic expression of the type I insulin-like growth factor receptor (IGF-IR) in mammary epithelial cells of MTB-IGFIR transgenic mice while RSTs resulted following the downregulation of the IGF-IR transgene in PMTs. As described in Franks et al. [31], the most differentially expressed gene between wild type mammary tissue and primary mammary tumors was Spp1 or OPN and this was confirmed by qRT-PCR. Raw and normalized gene expression data is available in the Gene Expression Omnibus (GEO:GSE32152).

Figure 1 shows the range of Spp 1 expression in $8 \mathrm{WT}$, 12 PMT and 8 RST samples as determined by the DNA microarray. Each of these tissues/tumors were obtained from different mice. The WT samples consistently expressed only very low levels of Spp1 while Spp1 expression in the tumor samples was more variable. Table 1 shows the average Spp1 expression as determined by the cDNA microarray. Quantitative RT-PCR was also performed on 4 WT, 4 PMT and 4 RST samples. As shown in Table 1, although the values were higher for each group, qRT-PCR confirmed the cDNA microarray data in that the PMTs had the highest level of Spp1 with the RSTs having less Spp1 than the PMTs but more than the WT samples which only expressed very low levels of Spp1.

OPN was also evaluated in PMTs and RSTs using immunohistochemistry. As shown in Fig. 2, OPN protein in PMTs was primarily expressed by stromal cells surrounding the tumor cells with only low, sporadic staining of OPN protein being observed in the tumor cells themselves (Fig. 2a, b). In contrast, high levels of OPN protein were frequently found in the tumor cells of RSTs (Fig. 2c, d). Sections lacking the primary antibody did not display any staining (data not shown).

\section{OPN expression in murine mammary tumor cell lines}

To determine whether the tumor cells themselves were producing OPN, cell lines derived from different MTBIGFIR mammary tumors were evaluated. The two best characterized lines are RJ345 cells (which have characteristics similar to PMT tumors in MTB-IGFIR transgenic mice) and RJ348 cells (which have characteristics similar to RST tumors in MTB-IGFIR transgenic mice) $[34,38-40]$. As shown in Table 2, RJ348 cells expressed higher levels of Spp1 than RJ345 cells. To confirm that murine mammary tumor cells with characteristics of human claudin-low breast cancer [34] express high levels of Spp1, another cell line, RM11A, which was derived from a different RST tumor was also examined. As shown in Table 2, RM11A cells expressed higher levels of Spp1 than the RJ345 cells. All the tumor cell lines expressed higher levels of Spp1 than the wild-type mammary tissue but lower levels than the PMTs (Table 1). Since RM11A cells expressed the highest level of Spp1 we collected conditioned media from these cells and

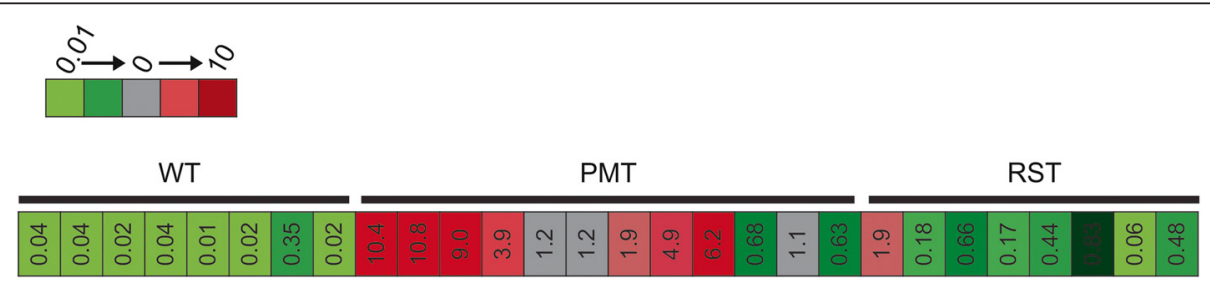

Fig. 1 Heat map illustrating the expression of OPN in 8 wild type mammary glands (WT), 12 primary mammary tumors (PMT) and 8 recurrent spindle tumors (RST) that arose in MTB-IGFIR transgenic mice as determined by CDNA microarray analysis. Numerical values in the box represent OPN expression relative to a universal mouse reference RNA sample. Microarrays were performed using Agilent Whole Mouse Genome $4 \times 44 \mathrm{k}$ Gene Expression Arrays (product number G4122F, Agilent) as described in [31] 
Table 1 OPN expression in tumors and tissue

\begin{tabular}{lcc}
\hline Sample & Spp1 (DNA Microarray) $)^{a}$ & Spp1 (qRT-PCR) $)^{b}$ \\
\hline WT & $0.07 \pm 0.04$ & $1.3 \pm 0.92$ \\
PMT & $4.33 \pm 1.13$ & $675.2 \pm 212.4$ \\
RST & $0.59 \pm 0.02$ & $94.7 \pm 48.7$
\end{tabular}

${ }^{a}$ OPN expression relative to a murine reference DNA sample, WT $(n=8)$, PMT $(n=12), \mathrm{RST}(n=8)$

${ }^{\mathrm{b}} \mathrm{OPN}$ expression relative to housekeeping genes Hprt and Ywhaz, WT $(n=4)$, PMT $(n=4)$, RST $(n=4)$

confirmed through western blotting that OPN was detectable in conditioned media of RM11A cells (data not shown).

\section{Spp1 knockdown decreases proliferation and increases apoptosis}

Since the claudin-low mammary tumor cell lines expressed higher levels of Spp1 and little is known about the function of OPN in claudin-low breast cancer, OPN function was evaluated in the claudin-low cell line RJ348. OPN was detected as two bands with molecular weights of $\sim 62 \mathrm{kDa}$ and $\sim 58 \mathrm{kDa}$ and three different siRNA oligonucleotides targeting different regions of OPN mRNA suppressed both the $62 \mathrm{kDa}$ and $58 \mathrm{kDa}$ OPN bands (Fig. 3a). A time course was then performed using OPN-siRNA ${ }^{2}$ in RJ348 cells and it was observed that OPN knockdown was achieved $48 \mathrm{~h}$ after RNAi treatment and OPN protein levels remained low $72 \mathrm{~h}$ after OPN RNAi treatment (Fig. 3b). OPN protein levels were reduced approximately $60 \%$ following OPN siRNA treatment. The efficacy of OPN-siRNA ${ }^{2}$ was confirmed in RM11A cells (Fig. 3c).

Proliferation was evaluated $48 \mathrm{~h}$ after OPN-siRNA ${ }^{2}$ transfection using immunofluorescence for Ki67. OPN knockdown produced a significant reduction in Ki67 positive staining in both RJ348 (Fig. 4a) and RM11A (Fig. 4b) cells compared to cells treated with the control siRNA. Proliferation was also evaluated using immunofluorescence for phosphorylated histone $\mathrm{H} 3$ and manual cell counting using trypan blue exclusion. Both of these experiments also showed a significant decrease in proliferation in RJ348 cells treated with OPN-siRNA ${ }^{2}$ compared to cells treated with the control siRNA (data not shown). As an alternative approach, RJ348 proliferation was assessed following incubation with $5 \mu \mathrm{g}$ of an OPN neutralizing antibody. As shown in Fig. 4c, treatment with an OPN neutralizing antibody also significantly reduced RJ348 proliferation, albeit to a lesser extent. Treating the RJ348 cells with $10 \mu \mathrm{g}$ of OPN neutralizing antibody resulted in a similar $20 \%$ reduction in cell proliferation (data not shown).

Apoptosis was evaluated $48 \mathrm{~h}$ after OPN-siRNA ${ }^{2}$ transfection using immunofluorescence for cleaved caspase 3. OPN knockdown produced a significant increase

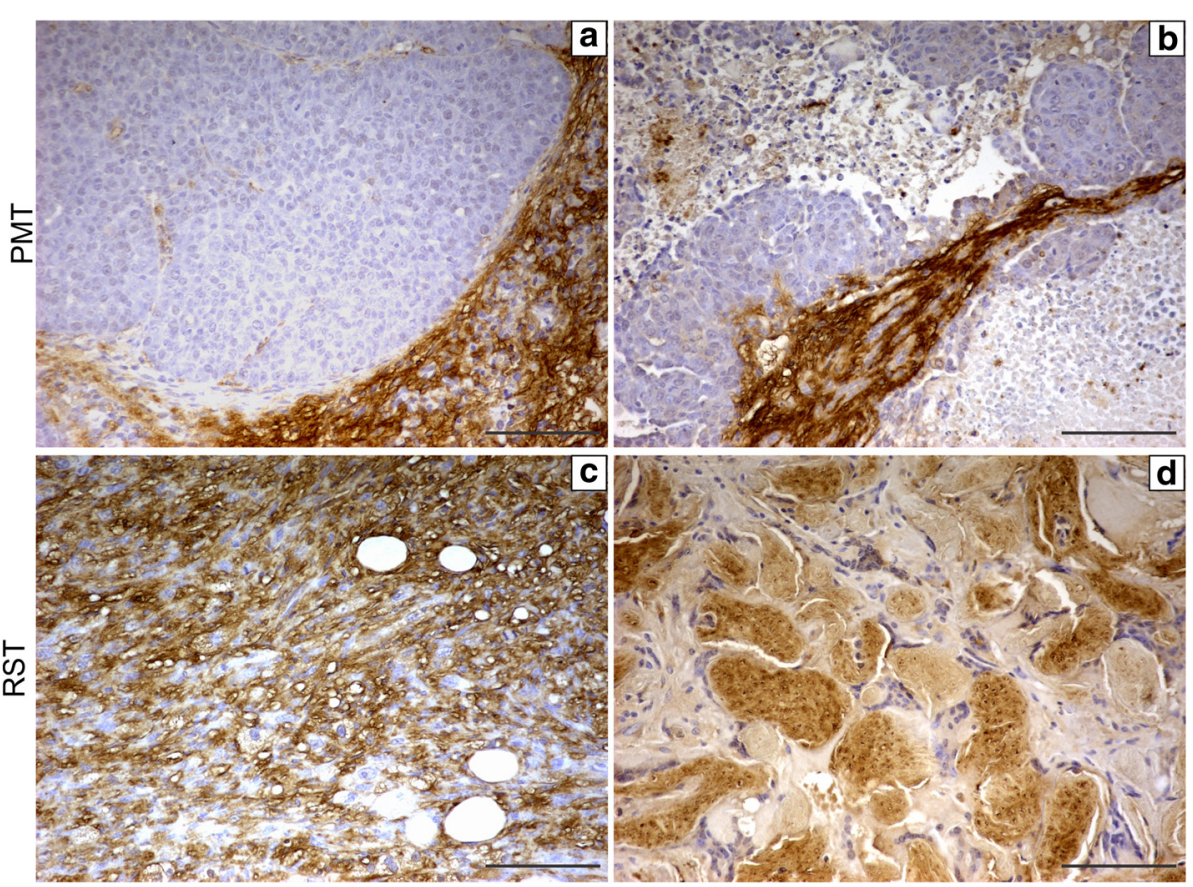

Fig. 2 Immunohistochemistry for OPN protein in (a, b) two independent PMT tumors and (c, d) two independent RST tumors. The brown staining indicates OPN protein which was expressed at only low levels in tumors cells of PMTs but at higher levels in the tumors cells or RSTs. RST tumors varied in histology with some tumors being composed primarily of spindle-shaped cells (c) and other tumors composed primarily of cells with squamous characteristics (d). Scale bars $100 \mu \mathrm{M}$ 
Table 2 OPN expression in mammary tumor cell lines

\begin{tabular}{lc}
\hline Sample & Spp1 (qRT-PCR) \\
\hline RJ348 & $21.8 \pm 5.7$ \\
RJ345 & $6.4 \pm 1.9$ \\
RM11A & $89.5 \pm 13.2$
\end{tabular}

${ }^{a}$ Spp1 expression relative to housekeeping genes Hprt and Ywhaz, RJ348 $(n=4), \operatorname{RJ} 345(n=4), \operatorname{RM} 11 \mathrm{~A}(n=4)$

in cleaved caspase 3 positive cells in both RJ348 (Fig. 4d) and RM11A (Fig. 4e) cells compared to cells treated with the control siRNA.

\section{Migration and invasion were reduced following OPN knockdown}

Cell migration was initially assessed using a scratch wound assay. RJ348 cells treated with OPN-siRNA ${ }^{2}$ had significantly impaired migration compared to RJ348 cells treated with the control siRNA at both the 24 and $48 \mathrm{~h}$ time points (Fig. 5a). Cell migration was also assessed using a transwell assay. Similar to the scratch wound assay, OPN knockdown significantly reduced RJ348 cell migration in the transwell assay (Fig. 5b-d). Scratch wound assays are difficult to perform using the RM11A cells as these cells have a disperse growth pattern and do not tightly pack together. Therefore migration and invasion was only evaluated in the RJ348 cells.

\section{Proliferation and migration following OPN receptor knockdown}

The two main receptors reported for OPN are CD44 and integrin $\alpha_{V} \beta_{3}$, however, other integrin receptors are also involved. Quantitative RT-PCR of Cd44, integrin $\alpha_{V}$ (Itgav), and integrin $\beta_{3}$ (Itgb3) mRNA expression revealed that only $C d 44$ and Itgav where highly expressed in RJ348 cells (Table 3). Similar levels of expression were observed in RM11A cells (Table 3).

To determine whether CD44 or integrin $\alpha_{V}$ were mediating the effects of OPN in RJ348 cells, these proteins were transiently knocked down using siRNA (RM11A cells were not evaluated). Quantitative RT-PCR revealed that $C d 44$ expression could be reduced to $12 \%$ of the control RNAi treated RJ348 cells (88 \% knockdown) while Itgav expression could be reduced to $32 \%$ of control RNAi treated RJ348 cells (68 \% knockdown).

Itgav knockdown significantly reduced proliferation in RJ348 cells (Fig. 6a) while Cd44 knockdown induced a small, non-significant reduction in RJ348 proliferation (Fig. 6c). With respect to migration, Cd44 knockdown did not significantly reduce wound closure (Fig. 6d). Itgav knockdown appeared to negatively impact cell adhesion in the scratch wound assay and thus only a transwell assay was performed. Itgav knockdown significantly reduce RJ348 transwell migration (Fig. 5b).
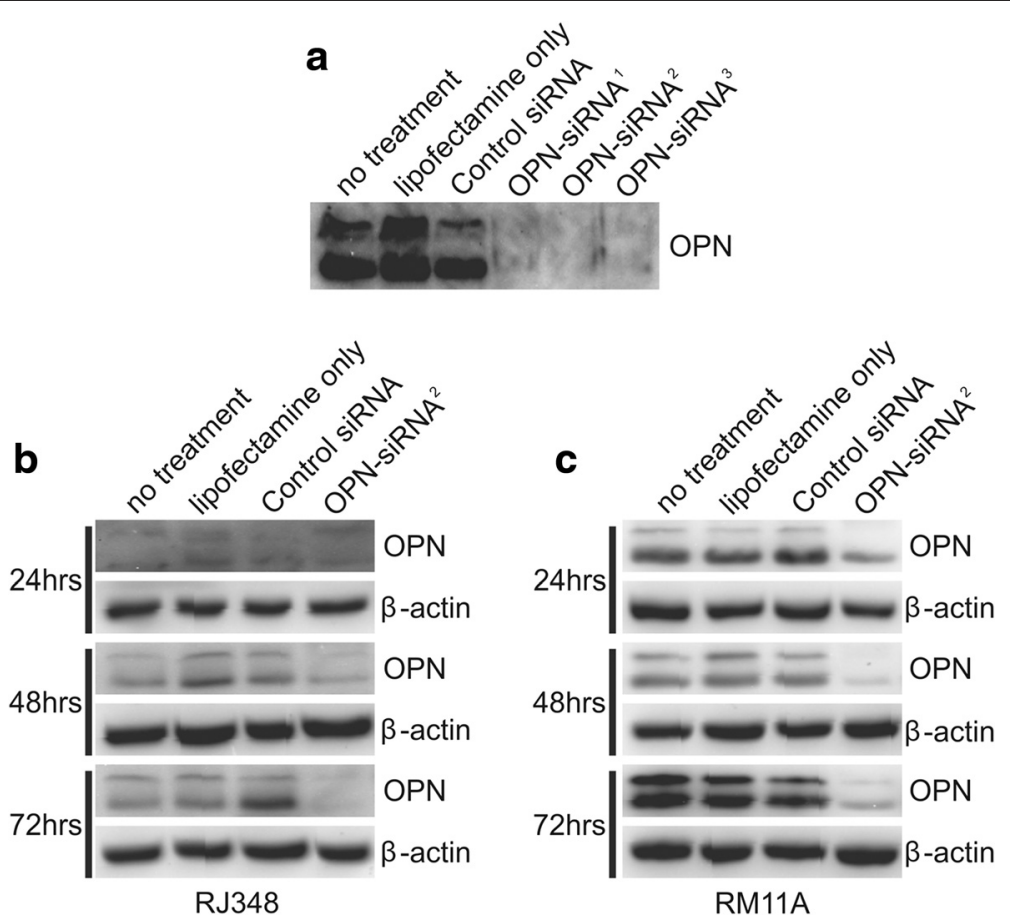

Fig. 3 Representative western blots showing OPN protein levels in (a) RM11A cell following no treatment, treatment with lipofectamine only, treatment with a GC control siRNA or 3 different siRNAs targeting different regions on OPN mRNA. Representative western blots of OPN protein level following no treatment, treatment with lipofectamine only, treatment with a GC control siRNA or treatment with OPN- siRNA ${ }^{2}$ in (b) RJ348 cells and (c) RM11A cells 24, 48 or $72 \mathrm{~h}$ after transfection 

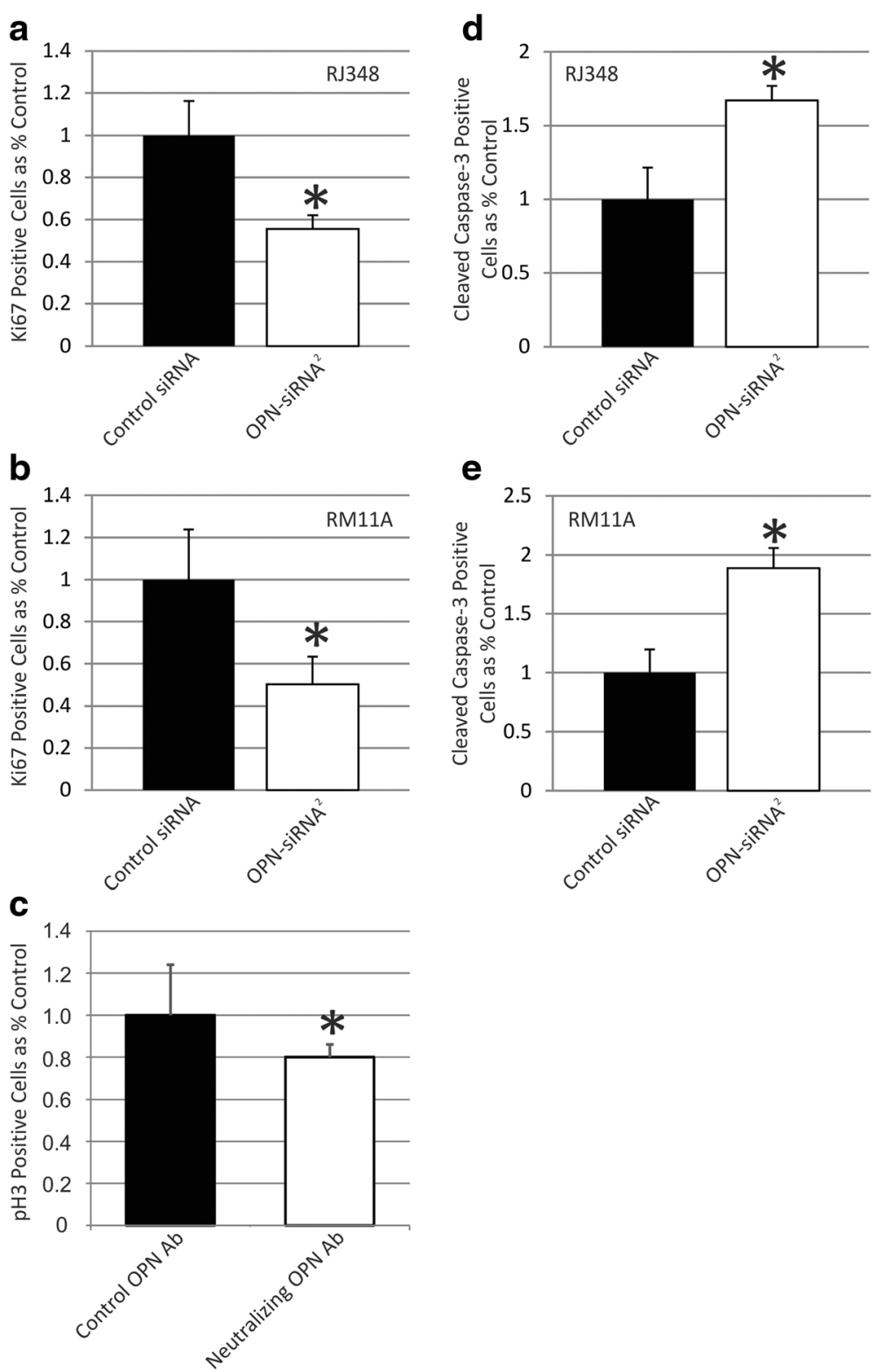

Fig. 4 Quantification of $(\mathbf{a}, \mathbf{b})$ Ki67 immunofluorescence or (d, e) cleaved caspase 3 immunofluorescence in (a, d) RJ348 cells or (B,E) RM11A cells after treatment with a GC control siRNA or OPN-siRNA ${ }^{2}$. The percentage of Ki67 positive or cleaved caspase 3 positive cells following OPN-siRNA ${ }^{2}$ treatment are presented relative to cells treated with the GC control siRNA. c phospho-histone H3 immunofluorescence in RJ348 cells following administration of $5 \mu \mathrm{g}$ of an OPN neutralizing antibody or $5 \mu \mathrm{g}$ of a control antibody. The percentage of phospho-histone $\mathrm{H} 3$ positive cells following OPN neutralizing antibody treatment is presented relative to the control antibody. A paired t-test was used to determine statistical significance, ${ }^{*} p^{<} 0.05, n \geq 3$

\section{Discussion}

Our lab has generated a transgenic model of mammary tumorigenesis. In this model, human IGF-IR is expressed in mammary epithelial cells in a doxycycline inducible manner and IGF-IR transgene expression induces mammary tumor development [30]. Down-regulation of IGFIR transgene (through doxycycline withdrawal) in established mammary tumors results in the regression of most of the mammary tumors and tumor recurrence in a subset of mice. Some of the recurrent mammary tumors acquire a spindle-like morphology and no longer express the IGF-IR transgene [33]. Gene expression analysis and clustering with human breast cancers revealed that the IGF-IR induced mammary tumors (also known as PMTs) express markers of luminal tumors but cluster closely with human basal-like tumors with the recurrent mammary tumors (also known as RSTs) express markers of claudin-low tumors and cluster closely with human 


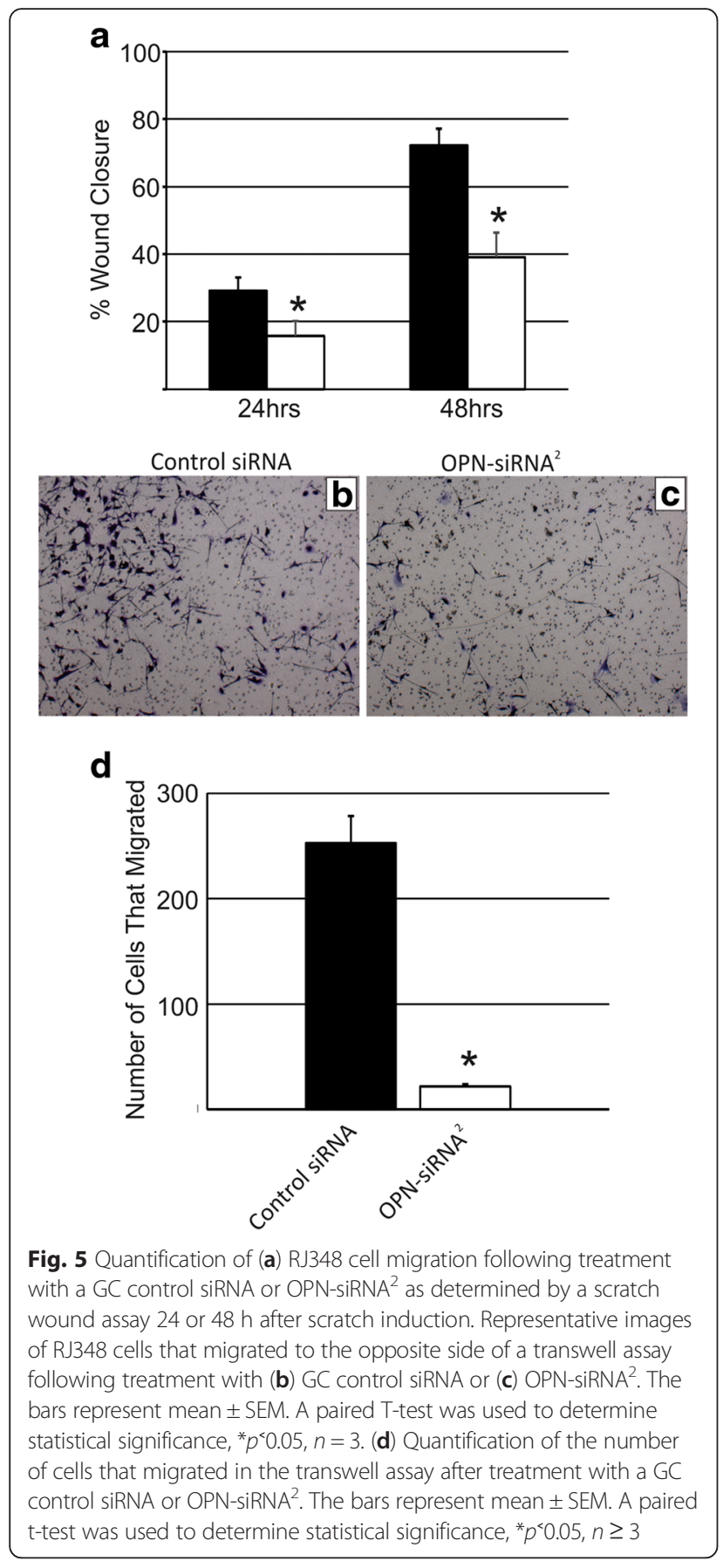

Table 3 Itgav, Itgb3 and C 444 expression in mammary tumor cell lines

\begin{tabular}{llll}
\hline & $\operatorname{ltgav}^{\mathrm{a}}$ & $\operatorname{ltgb3}^{\mathrm{a}}$ & Cd44 $^{\mathrm{a}}$ \\
\hline RJ348 & $0.92 \pm 0.14$ & $0.012 \pm 0.004$ & $0.99 \pm 0.12$ \\
RM11A & $0.91 \pm 0.11$ & $0.013 \pm 0.003$ & $0.91 \pm 0.11$ \\
\hline
\end{tabular}

${ }^{\mathrm{a} O P N}$ expression relative to housekeeping genes, Hprt and Ywhaz claudin low breast cancers [31]. Claudin-low mammary tumors are a subset of basal-like breast cancers that are typically estrogen receptor, progesterone receptor and HER2 negative, express low levels of claudins 2, 4 and 7 and have characteristics of progenitor cells [41-43]. Since the most differentially expressed gene between normal mouse mammary tissue and IGF-IR induced mammary tumors identified in our previous study was Spp1, this gene was further examined in this current manuscript in the transgenic model and cell lines derived from the IGF-IR transgenic mice (MTB-IGFIR transgenic mice).

Using cell lines derived from a PMT or two different RSTs from the MTB-IGFIR transgenic mice we found that the RST-derived cell lines (RJ348 and RM11A) had higher expression of OPN than the PMT-derived cell line (RJ345). This finding was somewhat surprising considering that PMTs in MTB-IGFIR transgenic mice had higher OPN expression than RSTs and may suggest that most of the OPN in the PMTs is produced by nontumor cells in the tumor microenvironment while the tumor cells themselves are the main source of OPN in the RSTs.

Immunohistochemical staining for OPN supported this theory as positive OPN staining in tumor cells from PMTs was typically low and sporadic, however, intense OPN staining was observed in stromal cells surrounding PMTs. In contrast, tumor cells in RSTs more frequently stained positive for OPN protein than tumor cells of PMTs. Studies in human breast cancer support this finding in that OPN was negatively correlated with luminal breast cancer subtypes [44] (no studies evaluating OPN expression in human claudin-low tumors have been described). Moreover, the human breast cancer cell line, MCF-7, which possesses characteristics of luminal breast cancer express lower levels of OPN than the human claudin-low breast cancer cell line, MDA-MB-231 [45]. Therefore, luminal tumors may depend on OPN from the microenvironment while claudin-low tumors may produce OPN.

We focused on the murine claudin-low mammary tumor cell lines since (1) the claudin-low murine mammary tumor cells expressed high levels of OPN, (2) claudin-low tumors are poorly understood, and (3) claudin-low tumor do not respond well to conventional therapies and thus alternative therapeutic strategies for this type of tumor requires identification. Our findings demonstrate that claudin-low mammary tumor cells rely on OPN for proliferation, survival and migration as knockdown of OPN using siRNA inhibited proliferation and migration while increasing apoptosis. An OPN neutralizing antibody was also capable of significantly inhibiting RJ348 proliferation albeit, less efficiently than OPN knockdown (apoptosis was not evaluated). These 

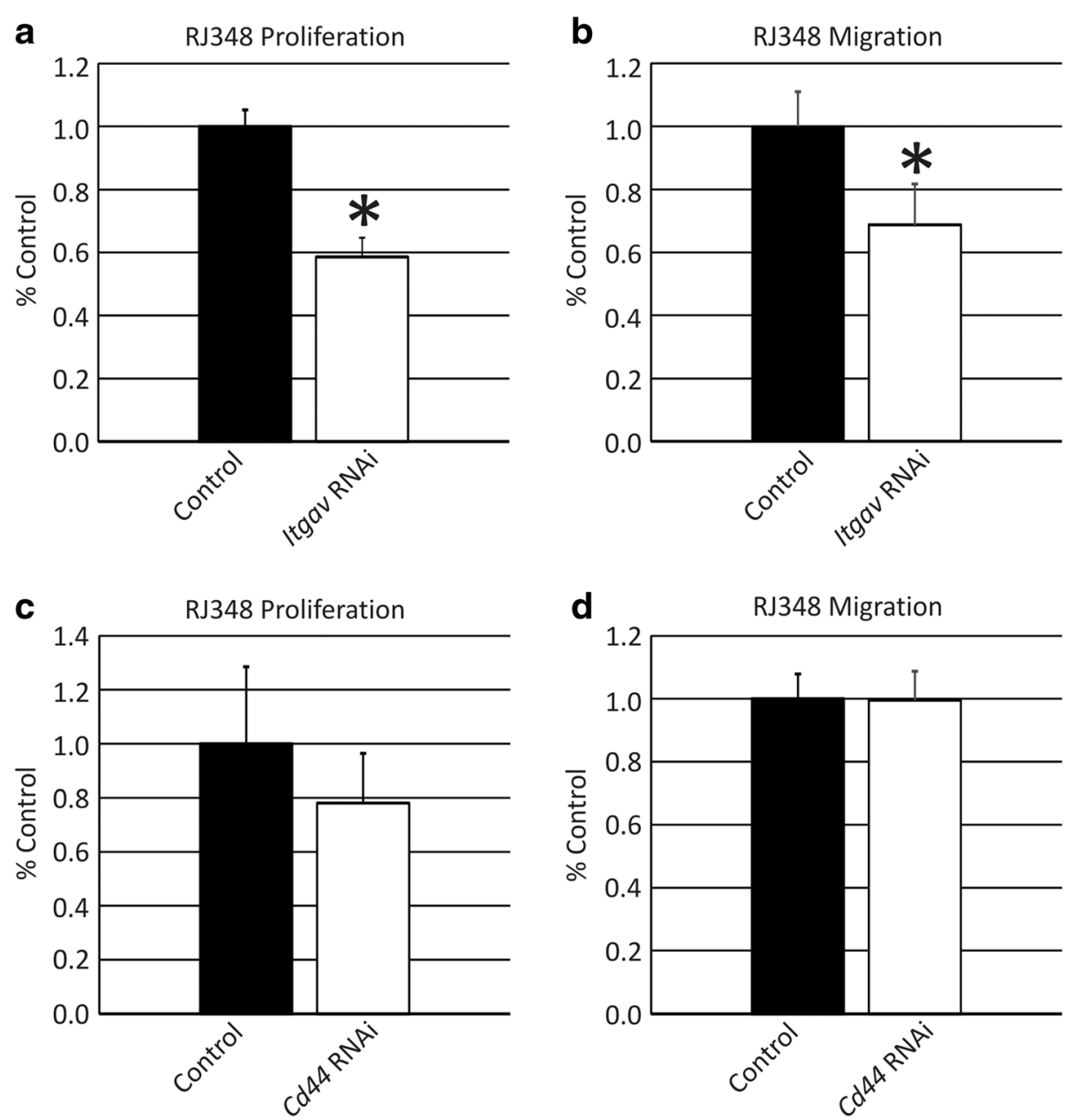

Fig. 6 Quantification of $(\mathbf{a}, \mathbf{c})$ phospho-histone $\mathrm{H} 3$ immunofluorescence, (b) transwell migration and (d) scratch wound closure in R3348 cells following treatment with $(\mathbf{a}, \mathbf{b})$ siRNA targeting Itgav or $(\mathbf{c}, \mathbf{d})$ siRNA targeting $C d 44$. Both proliferation and migration are presented relative to a GC control siRNA. * $p^{<} 0.05, n \geq 3$

findings are consistent with studies on MDA-MB-231 cells which showed that disruption of OPN function impaired proliferation [46, 47], survival [47-49] and migration $[46,48]$.

In an attempt to determine which receptors OPN was interacting with, the two best-characterized OPN receptors, CD44 and integrin $\alpha v \beta 3$ (ITGAV and ITGB3) [50] were examined. While RJ348 cells expressed considerable amounts of $C d 44$ and Itgav mRNA, only very low levels of Itgb3 were detected. To evaluate receptor function, $C d 44$ or Itgav mRNA was knocked down using siRNA. Only knockdown of Itgav and not Cd44 significantly decreased cell proliferation and migration suggesting that Itgav in association with a $\beta$-integrin, other than $\beta 3$, is mediating OPN's effects in RJ348 cells. OPN can also bind to $\alpha v$ containing integrins $\alpha v \beta 1, \alpha v \beta 5$, and $\alpha v \beta 6$ [8-10] and thus in RJ348 cells, OPN is likely mediating at least some of its effects via one of these integrin receptors. RNA sequencing has been performed on the RJ348 cells and Itgb1 and Itgb5, but not Itgb6 are highly expressed in RJ348 cells and thus presumably one of these two $\beta$-integrins are interacting with Itgav to mediate OPN signaling. The only published study that directly manipulated OPN receptors in human breast cancer utilized a CD44 neutralizing antibody in MDA-MB-231 and they observed that antibody mediated suppression of CD44 signaling inhibited migration [46]. Therefore, it remains unclear which receptors mediate the physiologic effects of OPN in claudin-low breast cancer.

\section{Conclusions}

Despite numerous studies implicating OPN in a variety of cancers, therapeutic strategies targeting OPN function have not materialized (OPN is being evaluated as a biomarker for a number of human cancers including non-small cell lung cancer, head and neck cancer, and pancreatic cancer (www.clinicaltrials.gov)). Our work, combined with published studies on MDA-MB-231 cells, argue that targeting OPN in claudin-low breast cancers may prove to be an effective therapeutic approach however a more complete 
understanding of the OPN-receptor interactions as well as development of potent/specific OPN inhibitors are required to translate this strategy from the pre-clinical to clinical setting.

\begin{abstract}
Abbreviations
Cd44, gene name for $\mathrm{Cd} 44$; CD44, protein name for Cd44; EGF, epidermal growth factor; GC, guanine-cytosine; IGF-IR, type I insulin-like growth factor receptor; Itgav, gene name for integrin $a_{v i} i T G A V$, protein name for integrin $a_{v i}$ Itgb3, gene name for integrin $\beta 3$; MTB-IGFIR, transgenic mouse expressing human IGF-IR in mammary epithelial cells in response to doxycycline; OPN, osteopontin protein; PMT, primary mammary tumor from MTB-IGFIR transgenic mouse; qRT-PCR, quantitative reverse transcription polymerase chain reaction; RJ345, murine mammary tumor cell line with luminal characteristics; RJ348, murine mammary tumor cell line with claudin-low characteristics; RM11A, murine mammary tumor cell line with claudin-low characteristics; RST, recurrent spindle tumor from MTB-IGFIR transgenic mouse; siRNA, small interfering ribonucleic acid; Spp1, gene name for osteopontin
\end{abstract}

\section{Funding}

This work was funded by a Canadian Institutes of Health Research (CIHR) operating grant (MOP\#106579) awarded to RAM. CIHR had no role in the study design, data collection, data analysis, data interpretation, the writing of the manuscript or the decision to submit this article for publication.

\section{Availability of data and materials}

Raw and normalized gene expression data from the DNA microarray analysis referred to in the results section is available in the Gene Expression Omnibus (GEO:GSE32152). Our cell lines have not been deposited in a repository but are available upon request (pending MTA approval).

\section{Authors' contributions}

SS performed the western blot experiments for the RJ348 cells including the siRNA knockdown, immunohistochemistry for Ki67 and cleaved caspase 3 in the RJ348 cells, the scratch wound assay in RJ348 cells and the transwell assay for the RJ348 cells. DT performed western blot experiments for the RM11A cells including the siRNA knockdown and immunohistochemistry for Ki67 and cleaved caspase 3 in the RM11A cells. JMcC performed three trials of RJ348 proliferation following treatment with the OPN neutralizing antibody and confirmed OPN knockdown using a second siRNA targeting OPN. PM performed two trials of RJ348 proliferation following treatment with the OPN neutralizing antibody. RM ran the project, extracted RNA for the DNA microarray experiment, performed all qRT-PCR experiments, as well as proliferation and migration following CD44 knockdown and wrote the manuscript. All authors read and approved the final manuscript.

\section{Competing interests}

The authors declare that they have no competing interests.

\section{Consent for publication}

No human tissues or samples were used in this study and thus details, images or videos related to individuals are not presented in this manuscript.

\section{Ethics approval and consent to participate}

Although animals were not used in this study, tissues originating from mice were used. These mice were housed and cared for following guidelines established by the Central Animal Facility at the University of Guelph and the guidelines established by the Canadian Council of Animal Care. Approval to use mice and collect tissue was granted by the Animal Care Committee at the University of Guelph. No human tissues or samples were used in this study.

Received: 6 August 2015 Accepted: 3 June 2016

\section{Published online: 10 June 2016}

\section{References}

1. Kunii Y, Niwa S, Hagiwara Y, Maeda M, Seitoh T, Suzuki T. The immunohistochemical expression profile of osteopontin in normal human tissues using two site-specific antibodies reveals a wide distribution of positive cells and extensive expression in the central and peripheral nervous systems. Med Mol Morphol. 2009;42(3):155-61.
2. Rangaswami H, Bulbule A, Kundu GC. Osteopontin: role in cell signaling and cancer progression. Trends Cell Biol. 2006;16(2):79-87.

3. Wai PY, Kuo PC. Osteopontin: regulation in tumor metastasis. Cancer Metastasis Rev. 2008;27(1):103-18.

4. Cho HJ, Cho HJ, Kim HS. Osteopontin: a multifunctional protein at the crossroads of inflammation, atherosclerosis, and vascular calcification. Curr Atheroscler Rep. 2009;11(3):206-13.

5. Sodek J, Ganss B, McKee MD. Osteopontin. Crit Rev Oral Biol Med. 2000; 11(3):279-303.

6. Tuck AB, Chambers AF, Allan AL. Osteopontin overexpression in breast cancer: knowledge gained and possible implications for clinical management. J Cell Biochem. 2007;102(4):859-68.

7. Weber GF, Lett GS, Haubein NC. Osteopontin is a marker for cancer aggressiveness and patient survival. Br J Cancer. 2010;103(6):861-9.

8. Denhardt DT, Giachelli CM, Rittling SR. Role of osteopontin in cellular signaling and toxicant injury. Annu Rev Pharmacol Toxicol. 2001;41:723-49.

9. Denhardt DT, Noda M, O'Regan AW, Pavlin D, Berman JS. Osteopontin as a means to cope with environmental insults: regulation of inflammation, tissue remodeling, and cell survival. J Clin Invest. 2001;107(9):1055-61.

10. Yokosaki Y, Matsuura N, Sasaki T, Murakami I, Schneider H, Higashiyama S, Saitoh Y, Yamakido M, Taooka Y, Sheppard D. The integrin alpha(9)beta(1) binds to a novel recognition sequence (SWYGLR) in the thrombin-cleaved amino-terminal fragment of osteopontin. J Biol Chem. 1999;274(51):36328-34.

11. Bellahcene A, Castronovo V. Increased expression of osteonectin and osteopontin, two bone matrix proteins, in human breast cancer. Am J Pathol. 1995;146(1):95-100.

12. Brown LF, Papadopoulos-Sergiou A, Berse B, Manseau EJ, Tognazzi K, Perruzzi CA, Dvorak HF, Senger DR. Osteopontin expression and distribution in human carcinomas. Am J Pathol. 1994;145(3):610-23.

13. Tuck AB, O'Malley FP, Singhal H, Harris JF, Tonkin KS, Kerkvliet N, Saad Z, Doig GS, Chambers AF. Osteopontin expression in a group of lymph node negative breast cancer patients. Int J Cancer. 1998;79(5):502-8.

14. Forootan SS, Foster CS, Aachi VR, Adamson J, Smith PH, Lin K, Ke Y. Prognostic significance of osteopontin expression in human prostate cancer. Int J Cancer. 2006;118(9):2255-61.

15. Hotte SJ, Winquist EW, Stitt L, Wilson SM, Chambers AF. Plasma osteopontin associations with survival and metastasis to bone in men with hormonerefractory prostate carcinoma. Cancer. 2002;95(3):506-12.

16. Agrawal D, Chen T, Irby R, Quackenbush J, Chambers AF, Szabo M, Cantor A, Coppola D, Yeatman TJ. Osteopontin identified as lead marker of colon cancer progression, using pooled sample expression profiling. J Natl Cancer Inst. 2002;94(7):513-21.

17. Bao LH, Sakaguchi H, Fujimoto J, Tamaya T. Osteopontin in metastatic lesions as a prognostic marker in ovarian cancers. J Biomed Sci. 2007;14(3):373-81.

18. Imano M, Satou T, Itoh T, Sakai K, Ishimaru E, Yasuda A, Peng YF, Shinkai M, Akai F, Yasuda T, et al. Immunohistochemical expression of osteopontin in gastric cancer. J Gastrointest Surg. 2009;13(9):1577-82.

19. Lin F, Li Y, Cao J, Fan S, Wen J, Zhu G, Du H, Liang Y. Overexpression of osteopontin in hepatocellular carcinoma and its relationships with metastasis, invasion of tumor cells. Mol Biol Rep. 2011;38(8):5205-10.

20. Ye QH, Qin LX, Forgues M, He P, Kim JW, Peng AC, Simon R, Li Y, Robles Al, Chen Y, et al. Predicting hepatitis B virus-positive metastatic hepatocellular carcinomas using gene expression profiling and supervised machine learning. Nat Med. 2003;9(4):416-23.

21. Chambers AF, Wilson SM, Kerkvliet N, O'Malley FP, Harris JF, Casson AG. Osteopontin expression in lung cancer. Lung Cancer. 1996;15(3):311-23.

22. Zhao B, Sun T, Meng F, Qu A, Li C, Shen H, Jin Y, Li W. Osteopontin as a potential biomarker of proliferation and invasiveness for lung cancer. J Cancer Res Clin Oncol. 2011;137(7):1061-70.

23. Anborgh PH, Mutrie JC, Tuck AB, Chambers AF. Role of the metastasispromoting protein osteopontin in the tumour microenvironment. J Cell Mol Med. 2010;14(8):2037-44.

24. Pietrowska M, Marczak L, Polanska J, Behrendt K, Nowicka E, Walaszczyk A, Chmura A, Deja R, Stobiecki M, Polanski A, et al. Mass spectrometry-based serum proteome pattern analysis in molecular diagnostics of early stage breast cancer. J Transl Med. 2009;7:60.

25. Shevde LA, Samant RS, Paik JC, Metge BJ, Chambers AF, Casey G, Frost AR, Welch DR. Osteopontin knockdown suppresses tumorigenicity of human metastatic breast carcinoma, MDA-MB-435. Clin Exp Metastasis. 2006;23(2):123-33.

26. Suzuki M, Mose E, Galloy C, Tarin D. Osteopontin gene expression determines spontaneous metastatic performance of orthotopic human breast cancer xenografts. Am J Pathol. 2007;171(2):682-92. 
27. Chakraborty G, Jain S, Patil TV, Kundu GC. Down-regulation of osteopontin attenuates breast tumour progression in vivo. J Cell Mol Med. 2008;12(6A): 2305-18.

28. Wang X, Chao L, Ma G, Chen L, Tian B, Zang Y, Sun J. Increased expression of osteopontin in patients with triple-negative breast cancer. Eur J Clin Invest. 2008;38(6):438-46.

29. Rodenburg W, Pennings JL, van Oostrom CT, Roodbergen M, Kuiper RV, Luijten M, de VA. Identification of breast cancer biomarkers in transgenic mouse models: A proteomics approach. Proteomics Clin Appl. 2010;4(6-7):603-12.

30. Jones RA, Campbell Cl, Gunther EJ, Chodosh LA, Petrik JJ, Khokha R, Moorehead RA. Transgenic overexpression of IGF-IR disrupts mammary ductal morphogenesis and induces tumor formation. Oncogene. 2007; 26(11):1636-44.

31. Franks SE, Campbell Cl, Barnett EF, Siwicky MD, Livingstone J, Cory S, Moorehead RA. Transgenic IGF-IR overexpression induces mammary tumors with basal-like characteristics while IGF-IR independent mammary tumors express a claudin-low gene signature. Oncogene. 2012;31(27):3298-309.

32. Jones RA, Moorehead RA. The impact of transgenic IGF-IR overexpression on mammary development and tumorigenesis. J Mammary Gland Biol Neoplasia. 2008:13(4):407-13.

33. Jones RA, Campbell Cl, Wood GA, Petrik JJ, Moorehead RA. Reversibility and recurrence of IGF-IR-induced mammary tumors. Oncogene. 2009;13:407-13.

34. Campbell Cl, Thompson DE, Siwicky MD, Moorehead RA. Murine mammary tumor cells with a claudin-low genotype. Cancer Cell Int. 2011;11:28.

35. Jones RA, Campbell Cl, Petrik JJ, Moorehead RA. Characterization of a novel primary mammary tumor cell line reveals that cyclin D1 is regulated by the type I insulin-like growth factor receptor. Mol Cancer Res. 2008;6(5):819-28.

36. Watson KL, Stalker L, Jones RA, Moorehead RA. High levels of dietary soy decrease mammary tumor latency and increase incidence in MTB-IGFIR transgenic mice. BMC Cancer. 2015;15:37.

37. Schneider CA, Rasband WS, Eliceiri KW. NIH Image to ImageJ: 25 years of image analysis. Nat Methods. 2012;9(7):671-5.

38. Thompson DE, Siwicky MD, Moorehead RA. Caveolin-1 expression is elevated in claudin-low mammary tumor cells. Cancer Cell Int. 2012;12:6.

39. Campbell $\mathrm{Cl}$, Moorehead RA. Mammary tumors that become independent of the type I insulin-like growth factor receptor express elevated levels of platelet-derived growth factor receptors. BMC Cancer. 2011:11:480.

40. Campbell Cl, Petrik JJ, Moorehead RA. ErbB2 enhances mammary tumorigenesis, oncogene-independent recurrence and metastasis in a model of IGF-IR-mediated mammary tumorigenesis. Mol Cancer. 2010;9:235.

41. Herschkowitz JI, Zhao W, Zhang M, Usary J, Murrow G, Edwards D, Knezevic J, Greene SB, Darr D, Troester MA, et al. Comparative oncogenomics identifies breast tumors enriched in functional tumor-initiating cells. Proc Natl Acad Sci USA. 2012;109(8):2778-83.

42. Herschkowitz JI, Simin K, Weigman VJ, Mikaelian I, Usary J, Hu Z, Rasmussen $K E$, Jones LP, Assefnia S, Chandrasekharan S, et al. Identification of conserved gene expression features between murine mammary carcinoma models and human breast tumors. Genome Biol. 2007;8(5):R76.

43. Prat A, Parker JS, Karginova O, Fan C, Livasy C, Herschkowitz JI, He X, Perou CM. Phenotypic and molecular characterization of the claudin-low intrinsic subtype of breast cancer. Breast Cancer Res. 2010;12(5):R68.

44. Ribeiro-Silva A, da Costa JP O. Osteopontin expression according to molecular profile of invasive breast cancer: a clinicopathological and immunohistochemical study. Int J Biol Markers. 2008;23(3):154-60.

45. Li NY, Weber CE, Mi Z, Wai PY, Cuevas BD, Kuo PC. Osteopontin upregulates critical epithelial-mesenchymal transition transcription factors to induce an aggressive breast cancer phenotype. J Am Coll Surg. 2013;217(1): 17-26. discussion 26.

46. Chu JE, Xia Y, Chin-Yee B, Goodale D, Croker AK, Allan AL. Lung-derived factors mediate breast cancer cell migration through CD44 receptor-ligand interactions in a novel ex vivo system for analysis of organ-specific soluble proteins. Neoplasia. 2014;16(2):180-91.

47. Yang L, Wei L, Zhao W, Wang X, Zheng G, Zheng M, Song X, Zuo W. Downregulation of osteopontin expression by RNA interference affects cell proliferation and chemotherapy sensitivity of breast cancer MDA-MB-231 cells. Mol Med Rep. 2012;5(2):373-6.

48. Zhang H, Guo M, Chen JH, Wang Z, Du XF, Liu PX, Li WH. Osteopontin knockdown inhibits alphav, beta3 integrin-induced cell migration and invasion and promotes apoptosis of breast cancer cells by inducing autophagy and inactivating the PI3K/Akt/mTOR pathway. Cell Physiol Biochem. 2014;33(4):991-1002.

49. Hahnel A, Wichmann H, Kappler M, Kotzsch M, Vordermark D, Taubert H, Bache M. Effects of osteopontin inhibition on radiosensitivity of MDA-MB231 breast cancer cells. Radiat Oncol. 2010;5:82.

50. Lu JG, Li Y, Li L, Kan X. Overexpression of osteopontin and integrin alphav in laryngeal and hypopharyngeal carcinomas associated with differentiation and metastasis. J Cancer Res Clin Oncol. 2011;137(11):1613-8.

\section{Submit your next manuscript to BioMed Central and we will help you at every step:}

- We accept pre-submission inquiries

- Our selector tool helps you to find the most relevant journal

- We provide round the clock customer support

- Convenient online submission

- Thorough peer review

- Inclusion in PubMed and all major indexing services

- Maximum visibility for your research

Submit your manuscript at www.biomedcentral.com/submit
Biomed Central 